\title{
Atuação do enfermeiro nos cuidados paliativos aos pacientes com câncer
}

\author{
Nurse's role in palliative care for cancer patients \\ El papel de la enfermera en los cuidados paliativos para pacientes con cáncer
}

Célia Mara Correa Nogueira ORCID: https://orcid.org/0000-0002-4100-2139 Centro Universitário IBMR, Brasil

E-mail: celiamaranogeira@gmail.com

Roberta da Silva Andrade Paschoal ORCID: https://orcid.org/0000-0003-1608-2138 Centro Universitário IBMR, Brasil E-mail: robertapaschoal1@gmail.com Claudia Regina Ferreira ORCID: https://orcid.org/0000-0003-3409-2081 Centro Universitário IBMR, Brasil E-mail: ferclaudia75@gmail.com

Michele de Souza Rodrigues ORCID: https://orcid.org/0000-0002-1808-7664 Centro Universitário IBMR, Brasil E-mail: michelly2827@hotmail.com Rafaela Lima de Oliveira ORCID: https://orcid.org/0000-0002-0994-3246 Centro Universitário IBMR, Brasil

E-mail: rafaela.limaoliveira@yahoo.com.br Luciano Godinho Almuinha Ramos ORCID: https://orcid.org/0000-0001-9958-3151 Centro Universitário IBMR, Brasil E-mail: lucianogodinho@yahoo.com.br

\begin{abstract}
Resumo
Este estudo disserta sobre a importância do enfermeiro nos cuidados paliativos dos pacientes oncológicos. Tem como objetivo: Destacar a importância da atuação do enfermeiro, junto aos pacientes oncológicos em cuidados paliativos. Para isso, foi utilizada a seguinte questão norteadora: Qual a importância do enfermeiro nos cuidados paliativos em pacientes com câncer? Como critérios de inclusão foram utilizados artigos científicos entre os anos de 2016 à 2021 , nas bases de dados, LILACS, BVS, SCIELO e PUBMED, em língua portuguesa, inglesa, francesa e espanhola. Como critérios de exclusão, foram retirados artigos fora do espaço temporal de pesquisa e que não abordavam a temática selecionada. Os resultados obtidos através dos 16 artigos científicos selecionados, trouxeram a efetivação da elaboração de três categorias temáticas: $O$ enfermeiro e os cuidados de enfermagem ao paciente com câncer, desafio do enfermeiro nos cuidados paliativos voltados aos portadores de câncer e manejo do enfermeiro na dor oncológica. O estudo concluiu que o enfermeiro é primordial no cuidado, mas encara desafios em diversos setores, tais como déficit na formação profissional, sobrecarga de trabalho, frustração e dificuldade de lidar com a morte. Este profissional, lidando diretamente com situações de sofrimento, além de todo desafio de sua carreira, pode ser atingido por conta do envolvimento emocional com o paciente.
\end{abstract}

Palavras-chave: Enfermeiro; Cuidados Paliativos; Pacientes; Câncer.

\begin{abstract}
This study discusses the importance of nurses in palliative care for cancer patients. Aims to: Highlight the importance of the role of nurses with cancer patients in palliative care. For this, the following guiding question was used: What is the importance of nurses in palliative care for cancer patients? As inclusion criteria, scientific articles were used between the years 2016 to 2021, in the databases, LILACS, BVS, SCIELO and PUBMED, in Portuguese, English, French and Spanish. As exclusion criteria, articles outside the research timeframe and which did not address the selected topic were removed. The results obtained through the 16 selected scientific articles brought about the realization of the elaboration of three thematic categories: The nurse and nursing care for patients with cancer, challenge for nurses in palliative care aimed at cancer patients and nurse management in cancer pain. The study concluded that nurses are essential in care, but face challenges in different sectors, such as deficits in professional training, work overload, frustration and difficulty in dealing with death. This professional, dealing directly with situations of suffering, in addition to every challenge in his career, can be reached because of his emotional involvement with the patient.
\end{abstract}

Keywords: Nurse; Palliative care; Patients; Cancer. 


\begin{abstract}
Resumen
Este estudio analiza la importancia de las enfermeras en los cuidados paliativos para los pacientes con cáncer. Tiene como objetivo: Resaltar la importancia del papel de las enfermeras con los pacientes con cáncer en los cuidados paliativos. Para ello, se utilizó la siguiente pregunta orientadora: ¿Cuál es la importancia de las enfermeras en los cuidados paliativos para los pacientes con cáncer? Como criterios de inclusión se utilizaron artículos científicos entre los años 2016 a 2021, en las bases de datos LILACS, BVS, SCIELO y PUBMED, en portugués, inglés, francés y español. Como criterio de exclusión se eliminaron los artículos fuera del plazo de investigación y que no abordaran el tema seleccionado. Los resultados obtenidos a través de los 16 artículos científicos seleccionados propiciaron la realización de la elaboración de tres categorías temáticas: La enfermera y la atención de enfermería al paciente con cáncer, desafío para enfermeras en cuidados paliativos dirigidos a pacientes oncológicos y manejo de enfermería en el dolor oncológico. El estudio concluyó que las enfermeras son fundamentales en la atención, pero enfrentan desafíos en diferentes sectores, como déficits en la formación profesional, sobrecarga de trabajo, frustración y dificultad para enfrentar la muerte. Este profesional, lidiando directamente con situaciones de sufrimiento, además de todos los retos de su carrera, puede ser alcanzado por su implicación emocional con el paciente.
\end{abstract}

Palabras clave: Enfermero; Cuidados paliativos; Pacientes; Cáncer.

\title{
1. Introdução
}

Segundo o Instituto Nacional do Câncer José Alencar Gomes da Silva, o câncer, dentre todas as patologias existentes, tem a segunda maior causa de mortalidade no Brasil e novos casos tem subido progressivamente, de acordo com as estatísticas de pesquisa. É um grave problema de saúde pública e, em nível mundial, a doença aumentou cerca de $20 \%$ nos últimos 10 anos. O ministério da saúde relata que apesar dos avanços na comunidade científica e dos tratamentos no século XX, fez com que grande parte das doenças se tornem crônica, aumentando a perspectiva de vida desses portadores, entretanto, no Brasil, dos 600 mil novos casos por ano, cerca de $60 \%$ são considerados em estado avançado e sendo encaminhado para tratamento paliativo. O conceito de cuidados paliativos foi redefinido em 2002 pela Organização Mundial em Saúde, e tem como objetivo melhorar a qualidade de vida dos pacientes e da sua rede de apoio, aliviar o sofrimento, tratar a sintomatologia física, social, psicológicos e espirituais, a partir da promoção do cuidado e identificação de forma precoce, avaliação e estabelecimento do tratamento.

A Organização Mundial em Saúde e o Instituto Nacional do Câncer José Alencar Gomes da Silva dizem que caso pacientes portadores de doença crônica, evolutiva e progressiva, que não possuem terapêutica curativa e que supostamente terá um período de vida pré-determinado, está elegível para os cuidados paliativos, este é encaminhado para uma equipe multiprofissional, composta por médicos, enfermeiros, nutricionistas, assistentes sociais, psicólogos, fonoaudiólogos e farmacêuticos, em prol do bem-estar de cada paciente ofertando as necessidades biopsicossociais de cada.

Em vista disso, o enfermeiro como membro da equipe multiprofissional paliativa, pode atuar das seguintes formas: plano terapêutico ou na gerência de cuidados, além de compreender as necessidades de cada paciente e família, com uma visão holística e humanizada aplicando a sistematização da assistência de enfermagem, a fim de organizar, planejar e implementar toda a operacionalização dos processos de acordo com cada procedimento, permitindo ao indivíduo o controle sobre sua vida e doença (Brasil, 2018).

Entretanto, Santos, et al., 2018 diz que o enfermeiro irá avaliar a qualidade de vida e conforto que está sendo ofertado ao paciente e tomará ações para que seja melhorado, vai introduzir métodos que diminuem o sofrimento de cada um relacionado ao tratamento e organizará medicamentos para alívio da dor sem ter como prioridade a cura, além disso pode-se desenvolver ações que incentivam amigos e familiares a estarem próximos até que o ciclo de vida desse paciente seja concluído, para que ele se sinta acolhido e amado. Percebe-se que os cuidados paliativos e o enfermeiro estão ligados diretamente, pois este é quem consagra a humanização e respeito ao paciente, que terá um final de vida confortável e sem dor (Matsumoto, 2012).

Diante disto, este estudo justifica-se em entender a importância do enfermeiro nos cuidados paliativos, visto que é a profissão no qual está ao lado do paciente em todos os momentos, desde o diagnóstico até a morte, tratando não só a sua saúde 
física, mas o emocional, espiritualidade inclusive aconselhamento e suporte ao luto, conforto, alívio dos sintomas e da dor. O enfermeiro é essencial no processo de organização do tratamento, promovendo a educação em saúde e orientações ao paciente e sua família.

Frente ao exposto, temos por objeto de estudo a importância do enfermeiro nos cuidados paliativos do paciente oncológico.

\section{Metodologia}

Para alcançar o objetivo proposto, foi utilizado uma revisão integrativa da literatura, sintetizando resultados obtidos em pesquisas sobre a temática de maneira sistemática, ordenada e abrangente. Para a seleção dos artigos foram acessadas as seguintes bases de dados: Biblioteca virtual em saúde (BVS) e (BVS) oncologia, Literatura Latino- Americana e do Caribe em Ciências da Saúde (LILACS), Scientific Electronic Library Online (SCIELO), Instituto Nacional do Câncer Jose Alencar Gomes da Silva (INCA) e National Library Of Medicine (PUBMED). O processo de desenvolvimento da pesquisa e seleção dos artigos foi conduzido por quatro pesquisadores independentes. No processo de montagem, foram encontrados 366 artigos, sendo 22 na BVS, 18 no LILACS, 29 no SCIELO e 296 PUBMED.

Foi escolhida a seguinte questão norteadora: Qual a importância do enfermeiro nos cuidados paliativos em pacientes com câncer? Com a busca dos estudos selecionados conforme os critérios de inclusão, foi utilizado artigos científicos entre os anos de 2016 à 2021, em português, artigos em sua versão na íntegra, artigos originais que englobassem os objetivos propostos e abordassem os descritores: Enfermeiro; Cuidados Paliativos; Pacientes, Câncer, totalizando 365 artigos, a busca dos descritores foi realizada na plataforma de Descritores em Ciências da Saúde, DECS com o objetivo de identificar os descritores em saúde para as pesquisas do artigos que serão trabalhados nesse artigo.

Conforme os critérios de exclusão, os artigos que não apresentavam a temática, artigos de revisão, artigos que não se relacionavam aos objetivos desta pesquisa, dissertações, teses e monografias foram descartados. Dessa forma foram excluídos um total de 351 artigos. Sendo que, após a leitura, foram selecionados 15 artigos para a elaboração final do estudo pelos bancos de dados.

Nas bases de dados BVS, LILACS, SCIELO E PUBMED, foi realizado um cruzamento dos descritores: Enfermeiro; Cuidados Paliativos; Pacientes; Câncer, foi empregado o operador boleano "AND", utilizando a filtragem para artigos em português, resumos, intervalo de anos proposto, artigos originais e análise de títulos, foram encontrados 366 artigos, sendo 350 excluídos por não abordar o tema proposto, ata superior há 5 anos, inacessibilidade e URLs não identificada. Neste caso, foi selecionado 15 artigos para a composição dessa pesquisa.

A seguir, um fluxograma apresentando como foi realizada a seleção dos artigos que compuseram a amostra final da revisão. 
Figura 1 - Fluxograma de seleção de artigos. Rio de Janeiro, RJ, Brasil, 2021.

\section{BASE DE DADOS}

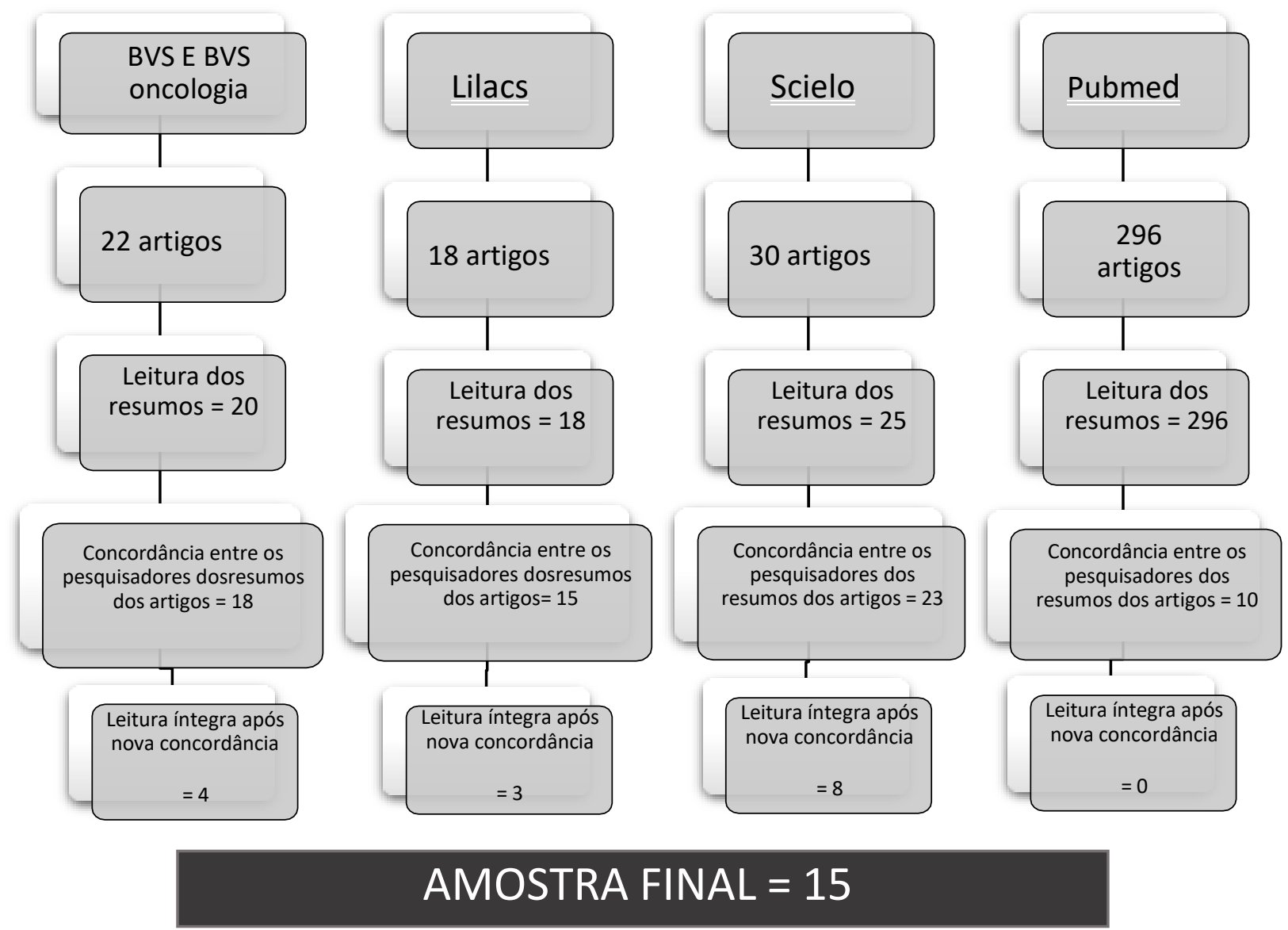

Fonte: Dados da pesquisa

Analisando a Figura 1, podemos observar os artigos que levaram a elaboração desta revisão, juntamente com sua ordem de busca, verificando, banco de dados, seleção, exclusão e escolha para a obtenção da amostra final. Foram excluídos artigos com data superior a 5 anos, relatos de experiências, artigos reflexivos, cartas ao editor e assuntos que não abordassem a temática proposta.

\section{Resultados e Discussão}

A busca dos artigos científicos foi realizada entre agosto e outubro de 2021, e para a apresentação das etapas de seleção dos artigos de acordo com as informações adquiridas, foi elaborado um quadro como metodologia com os principais dados dos artigos científicos escolhidos o qual está exposto a seguir. 
Quadro 1. Quadro expositivo dos artigos pesquisados neste estudo. Rio de Janeiro, RJ, Brasil, 2021.

\begin{tabular}{|c|c|c|}
\hline AUTOR/ANO & TÍTULO & OBJETIVOS \\
\hline $\begin{array}{l}\text { FERNANDES., } \\
\text { et al (2021) }\end{array}$ & $\begin{array}{l}\text { Conciliação medicamentosa em cuidados } \\
\text { paliativos oncológicos. }\end{array}$ & $\begin{array}{l}\text { Analisar o perfil das conciliações medicamentosasem pacientes que } \\
\text { estão sob cuidados paliativos oncológicos. }\end{array}$ \\
\hline $\begin{array}{l}\text { ANDRESS., et } \\
\text { al (2021) }\end{array}$ & $\begin{array}{l}\text { Assistência de enfermagem aos pacientes em } \\
\text { cuidados paliativos. }\end{array}$ & $\begin{array}{l}\text { Descrever o conhecimento do profissional enfermeiro na assistência } \\
\text { para melhoria da qualidade de vida, alívio da dor e sofrimento dos } \\
\text { pacientes em cuidados paliativos. }\end{array}$ \\
\hline $\begin{array}{l}\text { RODRIGUES., } \\
\text { et al (2020) }\end{array}$ & $\begin{array}{c}\text { Cuidados de enfermagem no manejo da dor em } \\
\text { pacientes adultos e idosos em cuidados } \\
\text { paliativos. }\end{array}$ & $\begin{array}{l}\text { Identificar os cuidados de enfermagem no manejo da dor de } \\
\text { pacientes adultos e idosos emcuidados paliativos. }\end{array}$ \\
\hline $\begin{array}{l}\text { ALMEIDA., et } \\
\text { al (2020) }\end{array}$ & $\begin{array}{l}\text { A relação entre oenfermeiro e o paciente nos } \\
\text { cuidados paliativosoncológicos }\end{array}$ & $\begin{array}{l}\text { Avaliar a relação e vivência do enfermeiro, relativo aos cuidados } \\
\text { com os pacientesoncológicos. }\end{array}$ \\
\hline $\begin{array}{l}\text { BORCHATT., } \\
\text { et al (2020) }\end{array}$ & $\begin{array}{c}\text { Avaliação das dimensõesda dor no paciente } \\
\text { oncológico }\end{array}$ & Mensurar a experiência dolorosa em pacientes oncológicos. \\
\hline $\begin{array}{l}\text { SILVA., et al } \\
\quad(2020)\end{array}$ & $\begin{array}{c}\text { Assistência deenfermagem a pacientes com } \\
\text { câncer em cuidadospaliativos: uma revisão } \\
\text { integrativa. }\end{array}$ & $\begin{array}{l}\text { Identificar na literatura quais sãoas evidências científicas sobre os } \\
\text { cuidados paliativos realizados pelo enfermeiro ao paciente com } \\
\text { câncer. }\end{array}$ \\
\hline $\begin{array}{l}\text { SIQUEIRA., et } \\
\text { al (2019) }\end{array}$ & $\begin{array}{l}\text { A atenção paliativaoncológica e suas } \\
\text { influências na percepção do enfermeiro }\end{array}$ & $\begin{array}{l}\text { Compreender quais são as principais influências psíquicas da } \\
\text { atenção paliativa oncológica na percepção do enfermeiro. }\end{array}$ \\
\hline $\begin{array}{l}\text { GOMES., et al } \\
\text { (2019) }\end{array}$ & $\begin{array}{c}\text { Cuidados paliativos:Relação eficaz entre } \\
\text { equipe de enfermagem,pacientes oncológicos e } \\
\text { seus familiares. }\end{array}$ & $\begin{array}{l}\text { Destacar a importância da comunicação na relação entre família, } \\
\text { equipe de enfermagem epacientes em finitude; ressaltar arelevância } \\
\text { dos familiares na habilidade e presteza da assistência estabelecida ao } \\
\text { doente oncológico em cuidados paliativos. }\end{array}$ \\
\hline $\begin{array}{l}\text { SANTOS., et al } \\
\text { (2018) }\end{array}$ & $\begin{array}{c}\text { Cuidados paliativos Prestados peloenfermeiro } \\
\text { ao paciente oncológico. }\end{array}$ & $\begin{array}{l}\text { Descrever os cuidados paliativosprestados pelo enfermeiro ao } \\
\text { paciente oncológico. }\end{array}$ \\
\hline $\begin{array}{l}\text { PICOLLO., et al } \\
\text { (2018) }\end{array}$ & $\begin{array}{c}\text { A atenção do enfermeiroao paciente em } \\
\text { cuidado paliativo. }\end{array}$ & $\begin{array}{l}\text { Conhecer a produção científica em relação a enfermagem acercados } \\
\text { cuidados paliativos; identificar o papel do enfermeirofrente aos } \\
\text { cuidados paliativos,elencar as principaiscompetências do profissional } \\
\text { e verificar a importância da equipe multidisciplinar. }\end{array}$ \\
\hline $\begin{array}{l}\text { HEY., et al } \\
(2017)\end{array}$ & $\begin{array}{l}\text { Participação daenfermeira nos cuidados } \\
\text { paliativos domiciliares. }\end{array}$ & $\begin{array}{l}\text { Descrever os cuidados paliativosdomiciliares realizados pela } \\
\text { enfermeira. }\end{array}$ \\
\hline $\begin{array}{l}\text { SCHIAVON., et } \\
\text { al (2016) }\end{array}$ & $\begin{array}{l}\text { Profissional de saúde frente a situação de ter } \\
\text { um familiar em cuidados paliativos por câncer. }\end{array}$ & $\begin{array}{l}\text { Conhecer a vivência do profissional de saúde na situaçãode ter um } \\
\text { familiar em cuidados paliativos por câncer. }\end{array}$ \\
\hline $\begin{array}{l}\text { EVANGELIST } \\
\text { A., et al (2016) }\end{array}$ & $\begin{array}{c}\text { Cuidados paliativos e espiritualidade: revisão } \\
\text { integrativa da literatura. }\end{array}$ & $\begin{array}{l}\text { Analisar artigos científicosdisseminados em periódicos on-line no } \\
\text { cenário internacionalacerca da temática cuidados paliativos e } \\
\text { espiritualidade. }\end{array}$ \\
\hline $\begin{array}{l}\text { LINDOLPHO., } \\
\text { et al (2016) }\end{array}$ & $\begin{array}{l}\text { Cuidados de enfermagem ao idoso nofim da } \\
\text { vida. }\end{array}$ & $\begin{array}{l}\text { Pontuar os parâmetros quenorteiam o cuidado de enfermagem ao } \\
\text { idoso, quevivencia sua terminalidade com uma visão existencialista. }\end{array}$ \\
\hline $\begin{array}{l}\text { SILVEIRA., et } \\
\text { al (2016) }\end{array}$ & $\begin{array}{l}\text { Cuidado paliativo eenfermeiros de terapia } \\
\text { intensiva: sentimentos que ficam. }\end{array}$ & $\begin{array}{l}\text { Conhecer os sentimentos dos enfermeiros acerca dos cuidados } \\
\text { paliativos em unidades de terapia intensiva de adultos. }\end{array}$ \\
\hline
\end{tabular}

Fonte: Dados da pesquisa.

Analisando o Quadro 1, podemos observar o esqueleto de organização e estrutura de seleção dos artigos, compondo o desenvolvimento desta revisão integrativa.

Dos 15 artigos selecionados no campo deste estudo, todos tiveram publicação entre 2016 e 2021, sendo em maior quantidade as apresentações dos artigos de 2016 e 2020.

Foi elaborado um gráfico com a porcentagem dos artigos encontrados com seus respectivos anos, a seguir podemos observá-lo na Figura 2 
Figura 2. Gráfico demonstrativo de porcentagem dos artigos encontrados no banco de dados entre os anos 2016 a 2021.

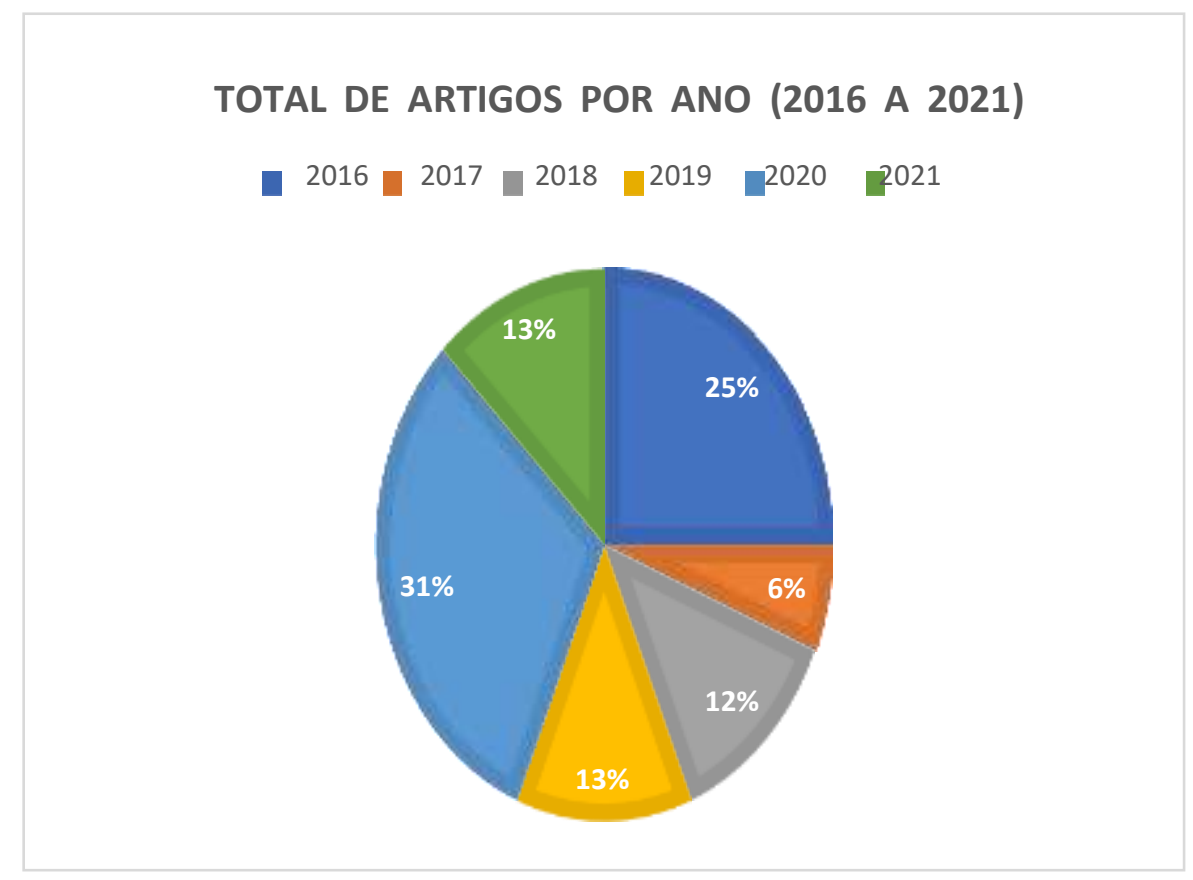

Fonte: Dados da pesquisa.

Os resultados obtidos através dos 15 artigos científicos selecionados, trouxeram efetivação a elaboração de três categorias temáticas: O enfermeiro e os cuidados de enfermagem ao paciente com câncer terminal; Desafios do enfermeiro nos cuidados voltados aos portadores de câncer e Manejo do enfermeiro na dor oncológica.

Categoria 1. O enfermeiro e os cuidados de enfermagem ao paciente com câncer terminal

O objetivo do enfermeiro ao paciente com câncer, não está baseado apenas em executar os procedimentos técnicos com a preocupação do cumprimento de princípios científicos e, sim de tratar o templo (corpo) de cada um, respeitando sua individualidade e por isso, se torna a profissão mais bela (Siqueira, et al., 2019).

Neste caso, é necessário um despertar para um cuidado de enfermagem intersubjetivo, pois estes cuidados são de pessoas envolvidas por emoções, sentimentos de medo, dor, perda, fragilização e até mesmo dificuldades socioeconômicas (Hey, et al., 2017).

Diante disso, Gomes, et al., 2019, demonstra que enfermeiro enquanto ciência do cuidado, utiliza-se de outras ciências para tomar decisões e ações e, desta forma, tudo que favorece ao cuidado do paciente e possui comprovação científica, se conceituam e fundamentam a ação deste profissional. Portanto, incluem e executam as teorias do cuidado, filosofia, psicologia, dentre outros para estruturar todas as suas decisões.

A literatura demonstra que o enfermeiro é de suma importância, pois este objetiva a pessoa nos cuidados paliativos e sua família a viver esses últimos momento o mais ativamente possível. Sempre afirmando a vida e demonstrando que a morte como parte do ciclo vital, não só do paciente, mas de todos os seres humanos. O enfermeiro tem um papel relevante, considerando que sua posição é privilegiada por permanecer a maior parte do tempo junto ao enfermo (Carvalho, 2009).

Segundo Lindolpho, et al., 2016, o enfermeiro deve observar o paciente de forma generalizada, com empatia e humanização, priorizando a terapêutica correta, a fim de promover afeto, carinho e atenção em cada paciente. É fundamental que os enfermeiros tenham a compreensão e a necessidade de dar uma importância no relacionamento terapêutico no sentido mais amplo. Sendo assim, o tratamento dentro da enfermagem oncológica consiste em emoções e sentimentos intensos e troca 
de ideias.

Entretanto, o enfermeiro deve-se atentar às necessidades do paciente, não somente físicas, mas psicológicas, espirituais e perfil socioeconômico para melhora no planejamento de cuidados através da redução de possíveis complicações. O fator principal é proporcionar uma qualidade de vida para a última fase da vida, amenizar o sofrimento proporcionando conforto e apoio.

Os estudos de Andres, et al., 2021, mostram que dentre as principais intervenções que o enfermeiro deve atuar, estão na redução dos sintomas, dentre eles, desidratação, constipação, fadiga, fraqueza, náusea, vômito, caquexia, infecção, anemia, alterações metabólicas e endócrinas, alterações na força muscular, dentre outras. Utiliza-se métodos não farmacológicos, com terapias não convencionais, destacando a aromaterapia, ludoterapia e toque terapêutico.

Categoria 2. Desafios do enfermeiro nos cuidados voltados aos portadores de câncer

O tema finitude, não é encarado com bons olhos pela maioria dos profissionais de saúde, a busca incansável pela cura, faz com que esses profissionais se sintam impotentes frente à morte iminente. Os profissionais recebem treinamentos para manter o paciente vivo, quando eles se deparam com uma pessoa portadora de doença crônica em fase avançada sem qualquer possibilidade de uma terapêutica curativa, os sentimentos de impotência e frustrações começam a fazer parte do cotidiano (Picollo, 2018).

Na literatura de Santos, et al., 2020, vimos que a forma mais eficaz no atendimento é a educação permanente de toda equipe. Para que o paciente e sua família sejam atendidos de forma completa, é necessário o entendimento por parte da equipe multidisciplinar sobre a vida e a história de cada um. Só assim, com uma equipe qualificada, é possível um resultado satisfatório.

$\mathrm{O}$ enfermeiro relata que iniciar uma abordagem multidisciplinar desde o diagnóstico de uma doença grave e sem possibilidades terapêuticas é o maior desafio para o profissional que desempenha o cuidado paliativo. Além disso, destaca-se que em muitos casos o profissional já se depara com pacientes em fase terminal, impossibilitando o desenvolvimento de estratégias que tem por objetivo a qualidade de vida, outro desafio é a dificuldade de singularizar, individualizar a assistência (Schiavon, et al., 2018).

Segundo Brandão, et al., 2017, mediante ao final da vida, os profissionais devem considerar os mecanismos de defesa do paciente e dos familiares, é fundamental valorizar e compreender os sentimentos dos que cuidam de pessoas em fase terminal. É preciso que toda equipe tenha participação em todas as fases do cuidado ao enfermo, no que consiste em orientá-lo sem coação demonstrando os benefícios e desvantagens de cada tratamento, de uma forma simplificada para que ele possa entender.

Para os enfermeiros o maior desafio foi controlar as emoções, pois lidar com a perda do paciente traz uma mistura de emoções, como raiva, frustação e impotência, outras influências são os enganos sobre a hospitalização e os aspectos que rodeiam, tais como doença, morte e cura que acabam interferindo no desenvolvimento pessoal e profissional dos que atuam em hospitais, visto que este é um local exclusivamente de cura (Silveira, et al., 2016).

Os estudos de Almeida, et al., 2020, demonstram que a maior dificuldade enfrentadas, são déficits na formação profissional, principalmente na questão de lidar com o processo de morrer e morte, noticiar o familiar, que evidencia que deve haver uma capacitação para os enfermeiros, a fim de melhorar essa assistência; falta de recursos; materiais e infraestrutura para atendimento. Outras dificuldades apontadas são: falta de medicamentos; desatualização ofertados pelo SUS; falta de insumos de laboratório; conscientização da população e aperfeiçoamento profissional. Portanto se faz necessário ações dentro das políticas públicas para melhoria da assistência prestada. 
Categoria 3. Manejo do enfermeiro na dor oncológica

A eficácia no tratamento da dor necessita de uma avaliação criteriosa de sua causa, entendendo os diferentes padrões de dor e conhecimento da melhor terapêutica. Uma boa avaliação inicial da dor vai definir uma linha de base para definição de intervenções posteriores. Um bom planejamento e parceria com cuidadores e a equipe de saúde, são vitais nesse processo (Rodrigues, et al., 2020).

Contudo, de acordo com Silva, et al., 2020, a avaliação da dor não é exata. Não há como mensurar a quantidade de dor de um paciente, quando é inviável estabelecer um padrão, uma vez que a mesma é subjetiva e individual. Para uma avaliação, o registro dos relatos e achados nos exames físicos devem seguir um roteiro para alcançar os objetivos para cessação da dor e unificar a linguagem da equipe, a mesma deve ser consensual e reavaliada trimestralmente até ser legitimada.

De acordo com a literatura de Borchatt, et al., 2020, essa avaliação deve ser realizada através de entrevista e exame físico, com o objetivo de identificar o tipo de dor nociceptiva, neuropática ou mista e compreender como isso afeta o indivíduo não somente na esfera física, mas também nas dimensões emocional, social e espiritual.

Mediante aos fatos, o preparo de enfermeiros é a principal estratégia para o controle da sintomatologia e dor prevalente em pacientes oncológicos avançados sob cuidados paliativos. Enfermeiros são profissionais que com mais frequência classificam a dor, avaliam a resposta do tratamento e analisa os efeitos colaterais, além de colaborar na reorganização do esquema analgésico, propõem nova farmacologia, prepara os pacientes e orientam os cuidadores após a alta hospitalar. Em algumas instituições que mantém acompanhamento em home care, os enfermeiros instituem, alteram e adequam o tratamento medicamentoso para controle das queixas álgicas (Evangelista, et al., 2016).

Em vista disso, de acordo com Fernandes, et al., 2020, a decisão dos fármacos, títulos, prescrição, doses de resgate são a sequência lógica necessária, todo planejamento deve ser de forma sistematizada e calculada, considerando todas as possibilidades dos sintomas, que em cuidados paliativos acontecem rapidamente e intensamente, a contínua observação e atenção às respostas e novas queixas, garantem o controle do processo analgésico.

O manual dos cuidados paliativos descreve que a intensidade da dor é um dos aspectos primordiais e deve ser continuamente reavaliada para verificar se a proposta está adequada ou não. Para medir a intensidade de forma rápida e segura, existem escalas validadas e que atendem à demanda. A instituição, por sua vez, deve ter base no perfil dos pacientes atendidos, adequados com sua realidade. Exemplos de escala: Visual analógica; visual numérica; descritores verbais; faces; PAINAD e behaviour pain scale (Carvalho, et al., 2009).

Este controle permite, uma decisão segura do processo sempre que necessário reiniciar, através da troca de fármacos, seguindo o rodízio de opiáceos preconizado e, novas prescrições e ou considerar o encaminhamento para procedimentos mais invasivos ou análise de refratariedade e intervenções mais radicais (Cardoso, et al.,2012).

Quando instituições mantém o acompanhamento em home care, os enfermeiros por exigências de resolutividade deste regime assistencial e, em benefício do paciente, mediante acordo com responsabilidades fundamentais e códigos do exercício profissional, é necessário uma capacitação, por meio de treinamento em serviço, para atuar como agentes diretos e ativos no controle da dor e das demais sintomatologias oncológicas, instituindo, alterando e adequando as terapias medicamentosas na modalidade assistencial de internação domiciliar, com respaldo institucional (Brasil, 2001).

\section{Considerações Finais}

Com base na pesquisa, destaca-se a importância do enfermeiro nos cuidados paliativos, pois a mesmo esta responsável na aplicação dos cuidados e bem-estar do paciente oncológico sem possibilidades de cura. Sua rotina de ofertar conforto ao paciente, além de favorecer o cliente, também proporciona aos familiares e cuidadores, fortalecendo todos os vínculos e a qualidade de vida de todos. 
Evidenciou-se, que o enfermeiro é primordial no cuidado, mas encara desafios em diversos setores que parecem estar longe de acabar, tais como déficit na formação profissional, de modo que ele precisa ter um conhecimento elevado para assistir integralmente este paciente e ainda oferecer o suporte aos familiares, sobrecarga de trabalho, frustração e dificuldade de lidar com a morte. Este profissional, lidando diretamente com situações de sofrimento, além de todo desafio de sua carreira, pode ser atingido por conta do envolvimento emocional com o paciente.

Como sugestão para pesquisas futuras, torna-se necessário a adaptação da prática em saúde oferecida pela equipe de enfermagem envolvida no cuidado do paciente diagnosticado e em tratamento do câncer, observou-se a necessidade de pesquisas nesse tema, criação de novos protocolos, melhoria nos insumos e medicamentos para o tratamento, conscientização da população e uma educação continuada para os profissionais, com a finalidade de obter a melhor assistência qualificada e eficaz ao paciente com câncer em cuidados paliativos.

Espera-se que esta pesquisa contribua com informações para um cuidado assistencial correto e a prática do enfermeiro no que tange os cuidados paliativos, para que a partir disso, implementações de melhoria no tratamento seja ofertado ao paciente terminal e que o profissional consiga desenvolver o seu trabalho de forma segura e eficaz.

\section{Referências}

Almeida et al. (2020). A relação entre o enfermeiro e o paciente nos cuidados paliativos oncológicos. Rev. Brazilian journals. 3 https://doi.org/10.34119/bjhrv3n2-011 https://www.brazilianjournals.com/index.php/BJHR/article/view/7394;

Andres et al. (2021). Assistência de enfermagem aos pacientes em cuidados paliativos. Research, Society and Development. https://rsdjournal.org/index.php/rsd/article/view/16140/14165;

Borchartt, et al. (2020). Avaliação das dimensões da dor no paciente oncológico. Revista Nursing. https://doi.org/10.36489/nursing.2020v23i266p4308-

Brandão et al. (2017). Cuidados paliativos do enfermeiro ao paciente oncológico. Rev ciencia em foco v24 http://revistas.uninorteac.com.br/index.php/DeCienciaemFoco0/article/view/147;

Brasil (2013). Ministério da Saúde. Secretaria de Atenção à Saúde. Departamento de Atenção Básica. Caderno de atenção domiciliar. Brasília: Ministério da Saúde. http://bvsms.saude. gov.br/bvs/publicacoes/inca/manual_cuidados_oncologicos.pdf.

Brasil (2015). Ministério da Saúde. Bases do tratamento do câncer. http://bvms.saude.gov.br/bvs/publicacoes/inca/acoes-cap6.pdf>

Brasil (2018). Resolução n ${ }^{\text {0 }}$ 41, de 31 de outubro de 2018. https://www.in.gov.br/materia/assetpublisher/Kujrw0TZC2Mb/content/id/51520746/do 1 -2018-1123-resolucao-n-41-de-31-de-outubro-de-2018-51520710

Cardoso et al. (2012). Classificação, fisiopatologia e avaliação da dor. Manual de Cuidados Paliativo. ANCP. (2a ed.), Sulina. $113-122$.

Carvalho. (2009). A dor do adoecer e do morrer. Bol. - Acad. Paul. Psicol., 29(2), 322-328, http://pepsic.bvsalud.org/scielo.php?script=sci_arttext\&pid=S1415- 711X2009000200009. Acesso em: 12 nov. 2021.

Evangelista et al. (2016). Cuidados Paliativos e espiritualidade: revisão integrativa da literatura. Rev. Bras. Enferm. 69 (3) •

Fernandes. (2020) Conciliação medicamentosa em cuidados paliativos oncológicos. Coleciona SUS. https://pesquisa.bvsalud.org/portal/resource/pt/biblio1150287 ;

Gomes et al. (2019). Cuidados paliativos: Relação eficaz entre equipe de enfermagem, pacientes oncológicos e seus familiares. Rev. Rede cuid. saúde. 3 http://publicacoes.unigranrio.edu.br/index.php/rcs/article/view/5522/3095

Hey et al. (2017). Participação da enfermeira nos cuidados paliativos domiciliares. REME rev. min. Enferm. v8 http://www.dx.doi.org/10.5935/14152762.20170010

http://www.revenf.bvs.br/scielo.php?script=sci_arttext\&pid=S1677- 38612016000200383

Instituto Nacional do Câncer José Alencar Gomes da Silva (2001). Cuidados paliativos oncológicos: controle da dor. http://www.inca.gov.br/publicacoes/manual_dor.pdf.

Instituto Nacional do Câncer José Alencar Gomes da Silva (2016). Coordenação de Prevenção e Vigilância. Estimativa: incidência de câncer no Brasil. Rio de Janeiro: INCA http://www.inca.gov.br/ bvscontrolecancer/publicacoes/Estimativa_2016.pdf

Lindolpho et al. (2016). Cuidados de enfermagem ao idoso no fim da vida. Rev. Ciênc. cuid. Saúde. 5(28) http://www.revenf.bvs.br/scielo.php?script=sci_arttext\&pid=S1677- 38612016000200383

Matsumoto. (2012). Cuidados Paliativos: conceitos, fundamentos e princípios. In: Manual de Cuidados Paliativos ANCP. Academia Nacional de Cuidados Paliativos. ANCP. www.paliativo.org.br/dl.php?bid=146 
Research, Society and Development, v. 10, n. 16, e576101624317, 2021

(CC BY 4.0) | ISSN 2525-3409 | DOI: http://dx.doi.org/10.33448/rsd-v10i16.24317

Organização Mundial de Saúde (2002). Definition of Palliative Care. Geneva: WHO, https://www.who.int/health- topics/palliative-care>.

Picollo et al. 2018. A atenção do enfermeiro ao paciente em cuidado paliativo. Rev Ciênc Med. 2018;27(2):85-92. http://dx.doi. org/10.24220/2318$0897 \mathrm{v} 27 \mathrm{n} 2 \mathrm{a} 3855$

Rodrigues et al., 2020. Cuidados de enfermagem no manejo da dor em pacientes adultos e idosos cuidados paliativos. Rev. Recom. 4(18) 10.19175 . http://seer.ufsj.edu.br/index.php/recom/article/view/3680/2544

Santos et al. (2017). Assistência Integral de Enfermagem aos pacientes em cuidados paliativos. Revista Saúde, 11(1), 36, 2017.

Santos et al. (2018). Cuidados paliativos prestados pelo enfermeiro ao paciente oncológico. Revista Dê Ciência em Foco. 13(8) http://revistas.uninorteac.com.br/index.php/DeCienciaemFoco0/article/view/147;

Schiavon et al. 2018. Profissional da saúde frente a situação de ter um familiar em cuidados paliativos por câncer. Rev.enf. atual. 5(9) http://dx.doi.org/10.1590/1983-1447.2016.01.55080;

Silva et al. (2020). Assistência de enfermagem a pacientes com câncer em cuidados paliativos. rev.enf. atual. 5(3)

Silveira et al. (2016). Cuidado paliativo e enfermeiros de terapia intensiva: sentimentos que ficam. Rev. Bras. Enferm. 69 (6) • https://doi.org/10.1590/00347167-2016-0267

Siqueira et al. (2019). A atenção paliativa oncológica e suas influências psíquicas na percepção do enfermeiro. REME rev. min. enferm. (43) (9) 10.5935/14152762.20190116 